\title{
THE BERNSTEIN INEQUALITY FOR SLICE REGULAR POLYNOMIALS
}

\author{
ZHENGHUA XU
}

\begin{abstract}
Due to the invalidation of the Gauss-Lucas type result for quaternionic polynomials, we first give in this paper an alternative proof of the Bernstein inequality in $L^{p}(1 \leq p \leq+\infty)$ for slice regular polynomials by the Fejér kernel and the Minkowski inequality. Secondly, we extend a result of Ankeny-Rivlin to the quaternionic setting via the Hopf lemma. By the way, some Turan inequalities are established for slice regular polynomials.
\end{abstract}

\section{INTRODUCTION}

Let $\mathbb{P}_{n}(\mathbb{C})$ be the class of complex polynomials $P(z)=\sum_{j=0}^{n} a_{j} z^{j}$, where $a_{j} \in \mathbb{C}, j=$ $0,1, \ldots, n$. For $P \in \mathbb{P}_{n}(\mathbb{C})$, define

$$
\|P\|_{p}:=\left(\frac{1}{2 \pi} \int_{0}^{2 \pi}\left|P\left(e^{i \theta}\right)\right|^{p} d \theta\right)^{\frac{1}{p}}, \quad 0<p<+\infty,
$$

and

$$
\|P\|_{\infty}:=\max _{|z|=1}|P(z)|=\max _{|z| \leq 1}|P(z)| .
$$

For any $P \in \mathbb{P}_{n}(\mathbb{C})$, it holds that

$$
\left\|P^{\prime}\right\|_{p} \leq n\|P\|_{p}, \quad 0<p \leq+\infty .
$$

The case $p=+\infty$ of (1.1) is known as the Bernstein inequality which is one of the most powerful tools with many important applications in approximation theory. It was proved by Zygmund [26] with the aid of an interpolation of Riesz for $1 \leq p<+\infty$ and by Arestov [2] by using the Jensen formula and the subharmonic function for $0<p<1$. For more extensions of the Bernstein inequality for complex polynomials, we refer to [4. Chapter 1], [7, Chapter 4], [17, Chapter 2], [18, Chapter 14] and [13.

Recently, the classical Bernstein inequality has been extended to the quaternionic setting depending heavily on the Gauss-Lucas type theorem and the structure of zero sets for quaternionic slice regular polynomials [8]. However, the method used in [8] is not valid for the octonionic and Clifford algebraic cases. What is worse, the alleged Gauss-Lucas type theorem given in [25] for quaternionic polynomials has not been verified since there exits a deadly mistake in [25, Proposition 3.14]. Very recently,

2010 Mathematics Subject Classification. 30C10, 41A17.

Key words and phrases. Bernstein inequality; Turan inequality; Quaternion.

This work was supported by the National Natural Science Foundation of China (No. 11801125) and the Fundamental Research Funds for the Central Universities (Nos. JZ2019HGTB0054 and JZ2018HGBZ0118). 
Ghiloni and Perotti have proved that the Gauss-Lucas type result in [25] is correct only for quaternionic polynomials of degree $d=2$ [12]. Hence, it is necessary to prove the Bernstein inequality for quaternions in other ways.

In this paper, we shall first give an alternative proof of the Bernstein inequality for quaternions in a more general version. Precisely speaking, we establish the analogy of (1.1) for $1 \leq p \leq+\infty$ in the setting of quaternions by applying the Fejér kernel and the Minkowski inequality, which is also applicable to both octonions and Clifford algebras. In fact, our method can be used to establish the case $1 \leq p \leq+\infty$ of (1.1) for complex polynomials with coefficients valued in real or complex normed linear spaces.

To state our results, let us recall some preliminary definitions and notation for quaternions.

Denote by $\mathbb{H}$ the non-commutative, associative, real algebra of quaternions with standard basis $\{1, i, j, k\}$, subject to the multiplication rules

$$
i^{2}=j^{2}=k^{2}=i j k=-1 \text {. }
$$

Every element $q=x_{0}+x_{1} i+x_{2} j+x_{3} k$ in $\mathbb{H}$ is composed by the real part $\operatorname{Re}(q)=x_{0}$ and the imaginary part $\operatorname{Im}(q)=x_{1} i+x_{2} j+x_{3} k$. The conjugate of $q \in \mathbb{H}$ is then $\bar{q}=\operatorname{Re}(q)-\operatorname{Im}(q)$ and its modulus is defined by $|q|=\sqrt{q \bar{q}}=\sqrt{x_{0}^{2}+x_{1}^{2}+x_{2}^{2}+x_{3}^{2}}$. The inverse of each nonzero element $q$ of $\mathbb{H}$ is given by $q^{-1}=|q|^{-2} \bar{q}$. Every $q \in \mathbb{H}$ can be expressed as $q=x+y I$, where $x, y \in \mathbb{R}$ and $I=\operatorname{Im}(q) /|\operatorname{Im}(q)|$ if $\operatorname{Im} q \neq 0$, otherwise we take $I$ arbitrarily such that $I^{2}=-1$. Here $I$ is an element of the unit 2 -sphere of purely imaginary quaternions,

$$
\mathbb{S}=\left\{q \in \mathbb{H}: q^{2}=-1\right\} .
$$

For every $I \in \mathbb{S}$, we denote by $\mathbb{C}_{I}$ the plane $\mathbb{R} \oplus I \mathbb{R}$, isomorphic to $\mathbb{C}$, and by $\mathbb{B}_{I}$ the intersection $\mathbb{B} \cap \mathbb{C}_{I}$, where $\mathbb{B}=\{q \in \mathbb{H}:|q|<1\}$ denotes the open unit ball of quaternions.

Throughout this paper, we consider the quaternionic polynomials in the form of

$$
P(q)=\sum_{j=0}^{n} q^{j} a_{j}, \quad a_{j} \in \mathbb{H}, j=0,1, \ldots, n .
$$

In fact, those polynomials are (left) slice regular functions in the sense of Gentili and Struppa [10]. For the slice regular polynomial $P(q)=\sum_{j=0}^{n} q^{j} a_{j}$, its derivative is defined as

$$
P^{\prime}(q)=\sum_{j=1}^{n} q^{j-1} j a_{j}
$$

Let $\mathbb{P}_{n}(\mathbb{H})$ be the class of slice regular polynomials $P(q)=\sum_{j=0}^{n} q^{j} a_{j}, a_{j} \in \mathbb{H}, j=$ $0,1, \ldots, n$. Let $P_{I}$ be the restriction of $P$ to $\mathbb{C}_{I}$. For $P \in \mathbb{P}_{n}(\mathbb{H})$, define

$$
\|P\|_{p}:=\sup _{I \in \mathbb{S}}\left(\frac{1}{2 \pi} \int_{0}^{2 \pi}\left|P_{I}\left(e^{I \theta}\right)\right|^{p} d \theta\right)^{\frac{1}{p}}, \quad 0<p<+\infty
$$

and

$$
\|P\|_{\infty}:=\max _{|q|=1}|P(q)|
$$


With the above notation, we can now present the first main result as follows.

Theorem 1.1. For $P \in \mathbb{P}_{n}(\mathbb{H})$ and $1 \leq p \leq+\infty$, we have

$$
\left\|P^{\prime}\right\|_{p} \leq n\|P\|_{p}
$$

The inequality is sharp. It becomes an equality for $P(q)=q^{n} a_{n}$ with $a_{n} \in \mathbb{H}$.

Remark 1.2. For complex polynomials, the Bernstein inequality in the statement of Theorem 1.1 is obtained, for example, by Corollary 14.6.4, p. 553 in the book [18, for all $0<p \leq+\infty$. The Bernstein inequality for quaternionic polynomials in the case when $0<p<1$ remains as an open question.

The refinement of the Bernstein inequality was conjectured by Erdös and proved by Lax [16] stating that

$$
\left\|P^{\prime}\right\|_{\infty} \leq \frac{n}{2}\|P\|_{\infty}
$$

for those complex polynomials $P \in \mathbb{P}_{n}(\mathbb{C})$ which have no zero in the open unit disk $\mathbb{D}=\{z \in \mathbb{C}:|z|<1\}$. See [3] and references therein for other proofs of this inequality.

As pointed out in [8, Theorem 3.1], the Erdös-Lax inequality (1.3) fails in the quaternionic setting in general and holds true for a subclass of the quaternionic polynomials as follows.

Proposition 1.3. Assume that $P \in \mathbb{P}_{n}(\mathbb{H})$ has no zero in the ball $\mathbb{B}$ and the zeros of $P$ are either spheres and/or real points and in addition to possibly up to one isolated zero $\alpha \in \mathbb{H} \backslash \mathbb{R}$ that has multiplicity 1 . Then

$$
\left\|P^{\prime}\right\|_{\infty} \leq \frac{n}{2}\|P\|_{\infty} .
$$

Using the convex combination identity for slice regular functions [19], we shall offer a brief method to establish the following version which contains the result in Proposition 1.3. Note that the proof of Proposition 1.4 is also valid for the octonion or Clifford algebra valued polynomials with all coefficients in a complex plane. In fact, we can find that the hypothesis in Proposition 1.4 that the slice regular polynomial preserves one slice can not be omitted in general as shown by the example $P(q)=q^{2}-q(i+j)+k$.

Proposition 1.4. Let $P \in \mathbb{P}_{n}(\mathbb{H})$. If $P_{I}$ has no zero in the open unit disk $\mathbb{B}_{I} \subset \mathbb{C}_{I}$ and $P_{I}\left(\mathbb{C}_{I}\right) \subset \mathbb{C}_{I}$ for some $I \in \mathbb{S}$, then

$$
\left\|P^{\prime}\right\|_{\infty} \leq \frac{n}{2}\|P\|_{\infty} .
$$

For the slice regular polynomial $P(q)=\sum_{j=0}^{n} q^{j} a_{j}$, denote $\widetilde{P}(q)=\sum_{j=0}^{n} q^{j} a_{n-j}=$ $q^{n} P\left(\frac{1}{q}\right)$. It follows that

$$
\max _{|q|=R}|P(q)|=R^{n} \max _{|q|=R}\left|q^{-n} P(q)\right|=R^{n} \max _{|q|=R}\left|\widetilde{P}\left(\frac{1}{q}\right)\right|=R^{n} \max _{|q|=1 / R}|\widetilde{P}(q)|,
$$

As in the complex case, we have, by the maximum modulus principle for slice regular functions [9, Theorem 7.1],

$$
\max _{|q|=1 / R}|\widetilde{P}(q)| \leq \max _{|q|=1}|\widetilde{P}(q)|=\max _{|q|=1}\left|P\left(\frac{1}{q}\right)\right|=\max _{|q|=1}|P(q)|, \quad R>1 .
$$


Hence,

$$
\max _{|q|=R}|P(q)| \leq R^{n} \max _{|q|=1}|P(q)|, \quad R>1 .
$$

We also establish the following result for $0<R<1$.

Theorem 1.5. For $P \in \mathbb{P}_{n}(\mathbb{H})$, we have

$$
\max _{|q|=R}|P(q)| \geq R^{n} \max _{|q|=1}|P(q)|, \quad 0<R<1 .
$$

As an application of the Erdös-Lax inequality, Ankeny and Rivlin strengthened the complex version of (1.4) as follows [1].

Theorem 1.6. Let $P \in \mathbb{P}_{n}(\mathbb{C})$ be such that $\|P\|_{\infty}=1$. If $P$ has no zero in $\mathbb{D}$, then

$$
\max _{|z|=R}|P(z)| \leq \frac{1+R^{n}}{2}, \quad R>1,
$$

with equality only for $P(z)=\left(\lambda+\mu z^{n}\right) / 2$, where $\lambda, \mu \in \partial \mathbb{D}$.

As pointed out in [1, the converse of Theorem 1.6 is false as shown by the example $P(z)=\left(z+\frac{1}{2}\right)(z+3)$. However, the following result for complex polynomials in the converse direction is valid. In fact, we can prove it in the quaternionic setting via the Hopf lemma, instead of the Gauss-Lucas theorem.

Theorem 1.7. Let $P \in \mathbb{P}_{n}(\mathbb{H})$ be such that $P(1)=\|P\|_{\infty}=1$, and

$$
\max _{|q|=R}|P(q)| \leq \frac{1+R^{n}}{2}, \quad 1<R<\delta+1,
$$

where $\delta$ is any positive number. Then $P$ does not have all its zeros within the open unit ball $\mathbb{B}$.

The remaining part of this paper is organized as follows. In Section 2, we shall prove Theorem 1.1 and then give some interesting remarks. For completeness, the proof of Proposition 1.4 is also given in Section 2. Finally, we establish Theorems 1.5 and 1.7 in Section 3. It is worth mentioning that Proposition 3.5 is vital to prove Theorems 1.5 and 1.7 due to the non-commutativity of quaternions.

\section{Proof of Theorem 1.1 And Proposition 1.4}

To prove Theorem 1.1, we resort to the Fejér kernel. The Fejér kernel is given by

$$
F_{n}(x)=\frac{1}{n+1} \sum_{k=0}^{n} D_{k}(x),
$$

where

$$
D_{k}(x)=\sum_{s=-k}^{k} \mathrm{e}^{i s x}
$$

is the $k$-th order Dirichlet kernel. 
It can also be written in a closed form as

$$
F_{n}(x)=\frac{1}{n+1}\left(\frac{\sin \frac{(n+1) x}{2}}{\sin \frac{x}{2}}\right)^{2}=\frac{1}{n+1} \frac{1-\cos (n+1) x}{1-\cos x} .
$$

The important feature of the Fejér kernel is the fact that it is non-negative. The Fejér kernel can also be expressed as

$$
F_{n}(x)=\sum_{j=-n}^{n}\left(1-\frac{|j|}{n+1}\right) e^{i j x} .
$$

Let $g: \mathbb{R} \rightarrow \mathbb{H}$ be continuous and $2 \pi$-periodic. Consider its Fourier series

$$
g(\theta)=\sum_{j=-\infty}^{+\infty} e^{i j \theta} c_{j}, \quad c_{j}=\frac{1}{2 \pi} \int_{0}^{2 \pi} e^{-i j \theta} g(\theta) d \theta .
$$

The $n$-th partial sum of the Fourier series is given by

$$
s_{n}(\theta ; g)=\sum_{j=-n}^{n} e^{i j \theta} c_{j}=\frac{1}{2 \pi} \int_{0}^{2 \pi} D_{n}(\theta-\varphi) g(\varphi) d \varphi
$$

and the corresponding $n$-th Cesàro sum has the expression

$$
\sigma_{n}(\theta ; g)=\frac{1}{n+1} \sum_{j=0}^{n} s_{j}(\theta ; g)=\frac{1}{2 \pi} \int_{0}^{2 \pi} F_{n}(\theta-\varphi) g(\varphi) d \varphi .
$$

From (2.1), we thus have

$$
\sigma_{n}(\theta ; g)=\sum_{j=-n}^{n}\left(1-\frac{|j|}{n+1}\right) e^{i j \theta} c_{j} .
$$

for any $g(\theta)=\sum_{j=-\infty}^{+\infty} e^{i j \theta} c_{j}$.

With above preliminaries, we come to give the proof of Theorem 1.1 .

Proof of Theorem 1.1. Starting from the slice regular polynomial $P(q)=\sum_{j=0}^{n} q^{j} a_{j}$, for any fixed $I \in \mathbb{S}$, we introduce the continuous and $2 \pi$-periodic function $g: \mathbb{R} \rightarrow \mathbb{H}$ via

It is evident that

$$
g(\theta)=e^{I n \theta} P\left(e^{-I \theta}\right) .
$$

$$
|g(\theta)|=\left|e^{I n \theta} P\left(e^{-I \theta}\right)\right|=\left|P\left(e^{-I \theta}\right)\right|
$$

and

$$
g(\theta)=\sum_{j=0}^{n} e^{I j \theta} a_{n-j}
$$

Notice that

$$
\left.\frac{1}{n} q^{-(n-1)} P^{\prime}(q)\right|_{q=e^{I \theta}}=\sum_{j=0}^{n} \frac{j}{n} e^{(j-n) I \theta} a_{j}=\sum_{j=0}^{n-1}\left(1-\frac{j}{n}\right) e^{-I j \theta} a_{n-j} .
$$


The right side is equal to $\sigma_{n-1}(-\theta ; g)$ due to (2.3).

From equality (2.2), it follows that

$$
\sigma_{n-1}(-\theta ; g)=\frac{1}{2 \pi} \int_{0}^{2 \pi} F_{n-1}(-\theta-\varphi) g(\varphi) d \varphi .
$$

Combing this with (2.6), we have

$$
\begin{aligned}
\left.\frac{1}{n}\left|q^{-(n-1)} P^{\prime}(q)\right|\right|_{q=e^{I \theta}} & \leq \frac{1}{2 \pi} \int_{0}^{2 \pi} F_{n-1}(-\theta-\varphi)|g(\varphi)| d \varphi \\
& =\frac{1}{2 \pi} \int_{0}^{2 \pi} F_{n-1}(\theta+\varphi)|g(\varphi)| d \varphi \\
& =\frac{1}{2 \pi} \int_{0}^{2 \pi} F_{n-1}(\varphi)|g(\varphi-\theta)| d \varphi,
\end{aligned}
$$

which implies that, by the Minkowski inequality for $1 \leq p<+\infty$ and (2.4),

$$
\begin{aligned}
\left(\int_{0}^{2 \pi}\left|\frac{P^{\prime}\left(e^{I \theta}\right)}{n}\right|^{p} \frac{d \theta}{2 \pi}\right)^{\frac{1}{p}} & \leq \int_{0}^{2 \pi} \frac{d \varphi}{2 \pi}\left(\int_{0}^{2 \pi} F_{n-1}^{p}(\varphi)|g(\varphi-\theta)|^{p} \frac{d \theta}{2 \pi}\right)^{\frac{1}{p}} \\
& =\int_{0}^{2 \pi} F_{n-1}(\varphi) \frac{d \varphi}{2 \pi}\left(\int_{0}^{2 \pi}|g(-\theta)|^{p} \frac{d \theta}{2 \pi}\right)^{\frac{1}{p}} \\
& =\left(\int_{0}^{2 \pi}\left|P\left(e^{I \theta}\right)\right|^{p} \frac{d \theta}{2 \pi}\right)^{\frac{1}{p}} .
\end{aligned}
$$

Hence,

$$
\left\|P^{\prime}\right\|_{p} \leq n\|P\|_{p}, \quad 1 \leq p<+\infty .
$$

From (2.4), (2.6) and (2.7), the case of $p=+\infty$ can be easily obtained. The proof is complete.

It is worth mentioning that the proof of Theorem 1.1 in the case $p=+\infty$ follows the method of [18, Theorem 14.1.1].

Two useful remarks concerning Theorem 1.1 are in order.

Remark 2.1. Denote by $\mathbb{R}_{0, m}$ the real Clifford algebra over $m$ imaginary units $e_{1}, e_{2}, \ldots, e_{m}$ which satisfy $e_{i} e_{j}+e_{j} e_{i}=-2 \delta_{i j}$. An element $a$ in $\mathbb{R}_{0, m}$ is denoted by $a=\sum_{A} a_{A} e_{A}$, where $a_{A} \in \mathbb{R}, A=h_{1} \ldots h_{r}, 1 \leq h_{1}<\ldots<h_{r} \leq m, e_{A}=e_{h_{1}} \ldots e_{h_{r}}$ and $e_{\emptyset}=1$. The modulus of $a$ is defined by $|a|=\left(\sum_{A}\left|a_{A}\right|^{2}\right)^{\frac{1}{2}}$. Note that the equality $|a b|=|a||b|$ does not hold generally for $a, b \in \mathbb{R}_{0, m}$ when $m \geq 3$. However, (2.4) still holds for Clifford algebras due to the following.

Lemma 2.2. ([14, Theorem 3.14]) For $a, b \in \mathbb{R}_{0, m}$ with $b \bar{b}=|b|^{2}$, we have

$$
|a b|=|a||b| \text {. }
$$

In particular,

$$
|x b|=|x||b|
$$

for any paravector $x=x_{0}+x_{1} e_{1}+\ldots+x_{m} e_{m} \in \mathbb{R}^{m+1}$. 
Hence, taking the same process as in Theorem 1.1, we can get the following Bernstein inequality in the Clifford algebra setting by the maximum modulus principle for slice monogenic functions [20, Theorem 3.1]. See [6] for the precise definition of slice monogenic functions.

Theorem 2.3. Let $P(x)=\sum_{j=0}^{n} x^{j} a_{j}: \mathbb{R}^{m+1} \rightarrow \mathbb{R}_{0, m}$ be a (left) slice monogenic polynomial of degree $n$ with Clifford algebraic coefficients $a_{n} \in \mathbb{R}_{0, m}$. Then

$$
\left\|P^{\prime}\right\| \leq n\|P\|
$$

where the norm of $P$ is defined by $\|P\|=\max _{|x| \leq 1}|P(x)|$.

Moreover, equality holds if and only if $P(x)=x^{n} a_{n}$ for some $a_{n} \in \mathbb{R}_{0, m}$.

Remark 2.4. In fact, the non-associative nature of octonions plays no role in (2.5) and (2.6) in the proof of Theorem 1.1 since Artin's theorem (cf. [23]) implies that the subalgebra generated by two elements in octonions is associative. Hence Theorem 1.1 still holds for octonionic slice regular polynomials in the sense of Gentili and Struppa in [11.

Now let us prove Proposition 1.4 .

Proof of Proposition 1.4. Let $P=\sum_{j=0}^{n} q^{j} a_{j}$ be the polynomial as described in the proposition. Notice that $P_{I}\left(\mathbb{C}_{I}\right) \subset \mathbb{C}_{I}$ for some $I \in \mathbb{S}$, equivalently, $a_{j} \in \mathbb{C}_{I}$ for $j=0,1, \ldots, n$, which implies that $P_{I}^{\prime}\left(\mathbb{C}_{I}\right) \subset \mathbb{C}_{I}$. The classical Erdös-Lax inequality applied to $P_{I}$ yields that

$$
\left\|P_{I}^{\prime}\right\| \leq \frac{n}{2}\left\|P_{I}\right\|
$$

where $\left\|P_{I}\right\|=\max _{q \in \partial \mathbb{B}_{I}}\left|P_{I}(q)\right|$.

Using the convex combination identity for slice regular functions [19], it holds that

$$
\left|P^{\prime}(\alpha+\beta J)\right|^{2}=\frac{1+\langle J, I\rangle}{2}\left|P^{\prime}(\alpha+\beta I)\right|^{2}+\frac{1-\langle J, I\rangle}{2}\left|P^{\prime}(\alpha-\beta I)\right|^{2},
$$

where $\langle\cdot, \cdot\rangle$ is Euclidean inner product in $\mathbb{R}^{3}$.

From (2.8) and (2.9), we have

$$
\left|P^{\prime}(\alpha+\beta J)\right| \leq \frac{n}{2}\left\|P_{I}\right\| \leq \frac{n}{2}\|P\|_{\infty}, \quad \forall \alpha+\beta J \in \mathbb{B}_{J}, \quad \forall J \in \mathbb{S},
$$

as desired.

\section{Proof of Theorems 1.5 and 1.7}

Now we recall some necessary definitions and properties from [5, 15, 9] in order to prove Theorem 1.7 .

Definition 3.1. Let $f, g: \mathbb{B} \rightarrow \mathbb{H}$ be two slice regular functions of the form

$$
f(q)=\sum_{n=0}^{\infty} q^{n} a_{n}, \quad g(q)=\sum_{n=0}^{\infty} q^{n} b_{n} .
$$


The regular product (or $*$-product) of $f$ and $g$ is the slice regular function defined by

$$
f * g(q)=\sum_{n=0}^{\infty} q^{n}\left(\sum_{k=0}^{n} a_{k} b_{n-k}\right) .
$$

Notice that the $*$-product is associative and is not, in general, commutative. Its connection with the usual pointwise product is clarified by the following result.

Proposition 3.2. Let $f$ and $g$ be slice regular on $\mathbb{B}$. Then for all $q \in \mathbb{B}$,

$$
f * g(q)= \begin{cases}f(q) g\left(f(q)^{-1} q f(q)\right) & \text { if } f(q) \neq 0 \\ 0 & \text { if } f(q)=0 .\end{cases}
$$

We remark that if $q=x+y I$ and $f(q) \neq 0$, then $f(q)^{-1} q f(q)$ has the same modulus and same real part as $q$. Therefore $f(q)^{-1} q f(q)$ lies in the same 2 -sphere $x+y \mathbb{S}$ as $q$. Notice that a zero $x_{0}+y_{0} I$ of the function $g$ is not necessarily a zero of $f * g$, but some element on the same sphere $x_{0}+y_{0} \mathbb{S}$ does. In particular, a real zero of $g$ is still a zero of $f * g$. To present a characterization of the structure of the zero set of a regular function $f$, we introduce the regular conjugate of $f$

$$
f^{c}(q)=\sum_{n=0}^{\infty} q^{n} \bar{a}_{n}
$$

and the symmetrization of $f$

$$
f^{s}(q)=f * f^{c}(q)=f^{c} * f(q)=\sum_{n=0}^{\infty} q^{n}\left(\sum_{k=0}^{n} a_{k} \bar{a}_{n-k}\right) .
$$

Proposition 3.3. Let $f$ be a slice regular function on $B(0, R)=\{q \in \mathbb{H}:|q|<R\}$ and choose $S=x+y \mathbb{S} \subset B(0, R)$. The zeros of $f$ in $S$ are in one-to-one correspondence with those of $f^{c}$. Furthermore, $f^{s}$ vanishes identically on $S$ if and only if $f^{s}$ has a zero in $S$, if and only if $f$ has a zero in $S$ (if and only if $f^{c}$ has a zero in $S$ ).

Remark 3.4. It is easy to see that $f^{c}(\cdot)=\overline{f(\cdot)}$ for any complex polynomial $f$. However, this result does not hold for slice regular polynomials.

Hence, we also need the following result to prove Theorems 1.5 and 1.7 .

Proposition 3.5. ([22, Proposition 3.2]) Let $f$ be a slice regular function on $B(0, R)$. For any sphere of the form $x+y \mathbb{S}$ contained in $B(0, R)$, the following equality holds

$$
\sup _{I \in \mathbb{S}}|f(x+y I)|=\sup _{I \in \mathbb{S}}\left|f^{c}(x+y I)\right| .
$$

Let us prove Theorem 1.5 .

Proof of theorem 1.5. Assume $P(q)=\sum_{j=0}^{n} q^{j} a_{j}$ and denote $Q(q)=\sum_{j=0}^{n} q^{j} \overline{a_{n-j}}=$ $q^{n} P^{c}\left(\frac{1}{q}\right)$. For $r>0$, we have, by Proposition 3.5.

$$
\max _{|q|=r}|Q(q)|=r^{n} \max _{|q|=r}\left|P^{c}\left(\frac{1}{q}\right)\right|=r^{n} \max _{|q|=r}\left|P\left(\frac{1}{q}\right)\right|=r^{n} \max _{|q|=1 / r}|P(q)| .
$$


Hence, by the maximum modulus principle for slice regular functions,

$$
\max _{|q|=R}|P(q)|=R^{n} \max _{|q|=1 / R}|Q(q)| \geq R^{n} \max _{|q|=1}|Q(q)|=R^{n} \max _{|q|=1}|P(q)|, \quad 0<R<1,
$$

as desired.

To establish the complex version of Theorem 1.7. Ankeny and Rivlin used the following lemma in terms of the Laguerre Theorem. Here we give an elementary proof for the sake of completeness.

Lemma 3.6. If

$$
P(z)=\left(z-z_{1}\right) \cdots\left(z-z_{n}\right)
$$

where $z_{m} \in \mathbb{D}=\{z \in \mathbb{C}:|z|<1\}$ for $m=1,2, \ldots, n$, then

$$
\left|\frac{P^{\prime}(a)}{P(a)}\right|>\frac{n}{2}, \quad \forall a \in \partial \mathbb{D} \text {. }
$$

Proof. Let $a=e^{i \theta}$ for some $\theta \in \mathbb{R}$. Then,

$$
\operatorname{Re} \frac{e^{i \theta}}{e^{i \theta}-z_{m}}>\frac{1}{2}
$$

for $z_{m} \in \mathbb{D}, m=1,2, \ldots, n$. Hence,

$$
\left|\frac{P^{\prime}(a)}{P(a)}\right| \geq \operatorname{Re}\left(e^{i \theta} \frac{P^{\prime}\left(e^{i \theta}\right)}{P\left(e^{i \theta}\right)}\right)=\sum_{m=1}^{n} \operatorname{Re} \frac{e^{i \theta}}{e^{i \theta}-z_{m}}>\frac{n}{2} .
$$

Now we can give the proof of Theorem 1.7

Proof of theorem 1.7. Let us consider the symmetrization $P^{s}$ of the quaternionic polynomial $P$. From Proposition 3.2 , it holds that

$$
P^{s}(q)= \begin{cases}P(q) P^{c}\left(P(q)^{-1} q P(q)\right) & \text { if } P(q) \neq 0 \\ 0 & \text { if } P(q)=0 .\end{cases}
$$

Hence

$$
\begin{aligned}
\max _{|q|=R}\left|P^{s}(q)\right| & =\max _{\{q \in \mathbb{H}:|q|=R, P(q) \neq 0\}}\left|P^{s}(q)\right| \\
& =\max _{\{q \in \mathbb{H}:|q|=R, P(q) \neq 0\}}\left|P(q) P^{c}\left(P(q)^{-1} q P(q)\right)\right| \\
& \leq \max _{|q|=R}|P(q)| \max _{\{q \in \mathbb{H}:|q|=R, P(q) \neq 0\}}\left|P^{c}\left(P(q)^{-1} q P(q)\right)\right| \\
& \leq \max _{|q|=R}|P(q)| \max _{|q|=R}\left|P^{c}(q)\right|,
\end{aligned}
$$

Combining this with Proposition 3.5, we have

$$
\max _{|q|=R}\left|P^{s}(q)\right| \leq \max _{|q|=R}|P(q)| \max _{|q|=R}\left|P^{c}(q)\right|=\max _{|q|=R}|P(q)|^{2},
$$

which implies that, by assumption,

$$
\left\|P^{s}\right\|_{\infty} \leq\|P\|_{\infty}^{2}=1
$$


and

$$
\max _{|q|=R}\left|P^{s}(q)\right| \leq\left(\frac{1+R^{n}}{2}\right)^{2} \leq \frac{1+R^{2 n}}{2}, \quad 1<R<\delta+1 .
$$

The condition $P(1)=1$ implies obviously that $P^{c}(1)=1$, and then, by (3.1),

$$
P^{s}(1)=1 \text {. }
$$

From (3.2) and (3.4), we see that $\left\|P^{s}\right\|_{\infty}=P^{s}(1)=1$. If $P^{s}$ is constant, then the claim is trivial. Otherwise, the Hopf lemma [21, Lemma 15.3.7] shows that $\left(P^{s}\right)^{\prime}(1)>0$. Then, given any $\epsilon>0$, sufficiently small, we have

$$
\left|P^{s}(1+\epsilon)-P^{s}(1)\right|=P^{s}(1+\epsilon)-1 \leq \frac{1+(1+\epsilon)^{2 n}}{2}-1 .
$$

Hence

$$
0<\left(P^{s}\right)^{\prime}(1) \leq n
$$

Suppose that $P$ has all its zeros in $\mathbb{B}$. From Proposition 3.3, we see that $P^{s}$ has all its zeros in $\mathbb{B}$. Noticing that $P^{s}$ is a quaternionic polynomial with real coefficients, we have, by (3.4) and Lemma 3.6.

$$
\left|\left(P^{s}\right)^{\prime}(1)\right|>n
$$

which contradicts inequality (3.5). Now the proof is complete.

An inverse inequality of (1.3) was proved by Turan [24, which says that

$$
\left\|P^{\prime}\right\|_{\infty} \geq \frac{n}{2}\|P\|_{\infty}
$$

for the complex polynomial $P \in \mathbb{P}_{n}(\mathbb{C})$ with all zeros in $\overline{\mathbb{D}}$.

From the proof of Theorem 1.7, we obtain the following Turan inequality.

Proposition 3.7. If $P \in \mathbb{P}_{n}(\mathbb{H})$ has all its zeros in $\overline{\mathbb{B}}$ and $P(1)=\|P\|_{\infty}$, then

$$
\left\|P^{\prime}\right\|_{\infty} \geq \frac{n}{2}\|P\|_{\infty} .
$$

Remark 3.8. For complex polynomials, the condition $P(1)=\|P\|_{\infty}$ in Proposition 3.7 is not essential. Indeed, we can consider the polynomial $Q(z)=P\left(z_{0} z\right) \overline{P\left(z_{0}\right)}$ when $\left|P\left(z_{0}\right)\right|=\|P\|_{\infty}$ for some $z_{0} \in \partial \mathbb{D}$. Furthermore, there are many quaternionic polynomials satisfying the statement of Proposition 3.7 such as $P(q)=\left(q^{2}+2 r q+\right.$ 1) $\left(q^{n-2} u+q^{n-3} u\right)$ for any $r \in[0,1]$, integer $n \geq 2$ and quaternion $u$. However, the construction of a concrete example of quaternionic polynomial of degree $d \geq 3$ with not all coefficients in the same plane and satisfying the hypothesis of Proposition 3.7 remains as an open question.

Proposition 3.9. If $P \in \mathbb{P}_{2}(\mathbb{H})$ has all its zeros in $\overline{\mathbb{B}}$, then

$$
\left\|P^{\prime}\right\|_{\infty} \geq\|P\|_{\infty}
$$


Proof. By assumption, the quaternionic polynomial $P \in \mathbb{P}_{2}(\mathbb{H})$ can be described as

$$
P(q)=(q-\alpha) *(q-\beta)=q^{2}-q(\alpha+\beta)+\alpha \beta,
$$

for some $\alpha, \beta \in \mathbb{H}$ with $|\alpha|,|\beta| \leq 1$. Then we obtain readily $\|P\|_{\infty} \leq 2+|\alpha+\beta|$ and $P^{\prime}(q)=2 q-(\alpha+\beta)$. Let us now show that

$$
\left\|P^{\prime}\right\|_{\infty}=\max _{|q|=1}\left|P^{\prime}(q)\right|=2+|\alpha+\beta| .
$$

If $\alpha+\beta=0$, this result is trivial. Otherwise, notice that $\left\|P^{\prime}\right\| \leq 2+|\alpha+\beta|$ and choose $q=-\frac{\alpha+\beta}{|\alpha+\beta|}$, as desired.

Acknowledgemants The main result of this work is part of the author's Ph.D. thesis. The author would like express his hearty thanks to his advisor, Professor Guangbin Ren, for helpful discussions. In addition, the author is very grateful to Professor Irene Sabadini for valuable communications and the anonymous referees for constructive suggestions which improve significantly the presentation of the paper.

\section{REFERENCES}

1. N. C. Ankeny, T. J. Rivlin, On a theorem of S. Bernstein, Pacific J. Math. 5 (1955), 849-852.

2. V. V. Arestov, On integral inequalities for trigonometric polynomials and their derivatives, Izv. Akad. Nauk SSSR Ser. Mat. 45 (1981) 3-22 (in Russian), English transl. in Math. USSR Izv. 18 (1982), 1-17.

3. A. Aziz, Q. G. Mohammad, Simple proof of a theorem of Erdös and Lax, Proc. Amer. Math. Soc. 80 (1980), no. 1, 119-122.

4. P. Borwein, T. Erdelyi, Polynomials and polynomial inequalities, Graduate Texts in Mathematics, 161. Springer-Verlag, New York, 1995.

5. F. Colombo, I. Sabadini, D. C. Struppa, Noncommutative Functional Calculus: Theory and Applications of Slice Hyperholomorphic Functions, Progress in Mathematics, 289, Birkhäuser/Springer Basel AG, Basel, (2011).

6. F. Colombo, I. Sabadini, D. C. Struppa, Slice monogenic functions, Israel J. Math. 171 (2009), 385-403.

7. R. A. DeVore, G. G. Lorentz, Constructive approximation, Grundlehren der Mathematischen Wissenschaften, 303. Springer-Verlag, Berlin, 1993.

8. S. G. Gal, I. Sabadini, On Bernstein and Erdös-Lax's inequalities for quaternionic polynomials, C. R. Math. Acad. Sci. Paris 353 (2015), no. 1, 5-9.

9. G. Gentili, C. Stoppato, D. C. Struppa, Regular functions of a quaternionic variable, Springer Monographs in Mathematics, Springer, Berlin-Heidelberg, 2013.

10. G. Gentili, D. C. Struppa, A new theory of regular functions of a quaternionic variable, Adv. Math. 216 (2007), no. 1, 279-301.

11. G. Gentili, D. C. Struppa, Regular functions on the space of Cayley numbers, Rocky Mountain J. Math. 40 (2010), 225-241.

12. R. Ghiloni, A. Perotti, The quaternionic Gauss-Lucas theorem, Ann. Mat. Pura Appl. (4) 197 (2018), no. 6, 1679-1686.

13. N. K. Govil, R. N. Mohapatra, Markov and Bernstein type inequalities for polynomials, J. of Inequal. and Appl., 3 (1999), 349-387.

14. K. Gürlebeck, K. Habetha, W. Sprößig, Holomorphic functions in the plane and ndimensional space, Birkhäuser Verlag, Basel, 2008.

15. T. Y. Lam, A first course in noncommutative rings. Graduate Texts in Mathematics, 131. SpringerVerlag, New York, 1991.

16. P. D. Lax, Proof of a conjecture of P. Erdös on the derivative of a polynomial, Bull. Amer. Math. Soc. 50 (1944), 509-513. 
17. M. Marden, Geometry of polynomials, Second edition. Mathematical Surveys, No. 3 American Mathematical Society, Providence, R.I. 1966.

18. Q. I. Rahman, G. Schmeisser, Analytic theory of polynomials, London Mathematical Society Monographs. New Series, 26. The Clarendon Press, Oxford University Press, Oxford, 2002.

19. G. Ren, X. Wang, Z. Xu, Slice regular functions on regular quadratic cones of real alternative algebras, Trends in Mathematics, Birkhäuser, (2016), 227-245.

20. G. Ren, Z. Xu, Schwarz's lemma for slice Clifford analysis, Adv. Appl. Clifford Algebr. 25 (2015), no. 4, 965-976.

21. W. Rudin, Function theory in the unit ball of $\mathbb{C}^{n}$, Reprint of the 1980 edition. Springer-Verlag, Berlin, 2008.

22. C. Della Rocchetta, G. Gentili, G. Sarfatti, A Bloch-Landau theorem for slice regular functions, Advances in hypercomplex analysis, 55-74, Springer INdAM Ser., 1, Milan, 2013.

23. R. D. Schafer, An introduction to nonassociative algebras, Pure and Applied Mathematics, Vol. 22 Academic Press, New York, 1966.

24. P. Turan, Über die Ableitung von Polynomen, Compositio Math. 7 (1939), 89-95.

25. F. Vlacci, The Gauss-Lucas theorem for regular quaternionic polynomials, Hypercomplex analysis and applications, 275-282, Trends Math., Birkhuser/Springer Basel AG, Basel, 2011.

26. A. Zygmund, A remark on conjugate series, Proc. London Math. Soc. 34 (1932), 392-400.

Zhenghua Xu, School of Mathematics, HeFei University of Technology, Hefei 230601, CHINA

E-mail address: zhxu@hfut.edu.cn 